\title{
Corticofugal projections from the anterior olfactory nucleus target olfactory bulb principal cells
}

\author{
Kurt R. Illig* \\ The anterior olfactory nucleus (AON) is a cortical structure reciprocally connected with the olfactory bulb \\ $(\mathrm{OB})$ and the piriform cortex. Here, using small extracellular and intracellular injections to trace the terminal \\ projections in the $O B$ from individual cells in the rat $A O N$, and examining the morphology and distribution of \\ these projections, I report that these projections appear to preferentially target and directly synapse with \\ mitral cells. These findings suggests that the AON acts to modulate incoming olfactory information based on \\ ongoing processing, perhaps in a way that tunes the OB to signal the presence of particular odors.
}

The anterior olfactory nucleus (AON) is a central olfactory cortical structure that has heavy reciprocal connections with both the olfactory bulb (OB) and the piriform cortex. The main part of the AON (pars principalis) is comprised of two layers: a superficial plexiform layer (layer I) consisting of axons of $\mathrm{OB}$ principal cells and of fibers from the contralateral $\mathrm{AON}$, and a dense cell body layer (layer II) consisting of pyramidal cell somata. Deep to layer II lies a poorly-defined plexiform layer consisting of corticofugal fibers from the piriform cortex and from the contralateral $\mathrm{AON}$, and of fibers containing modulatory neurotransmitters (e.g., acetylcholine and norepinephrine). ${ }^{1-3}$

It has long been known that cells in the AON project to the $\mathrm{OB}$, however previous experiments used large injections of tracer molecules that rendered a fine-scale analysis of these centrifugal projections impossible. The organization of these projections is of particular significance in light of recent studies suggesting a specific, point-to-point mapping displayed in projections from receptor cells in the olfactory epithelium to the $\mathrm{OB}$ and within the pars externa of the $\mathrm{AON}^{4}$. In this study, we have traced the terminal projections in the $\mathrm{OB}$ from individual cells in the rat $\mathrm{AON}$ using small extracellular injections of Phaseolus vulgaris leucoagglutinin and in vivo intracellular injections of biotinylated dextran amine (3000 MW). Here, we present a detailed analysis of the terminal processes (synaptic boutons) of these corticobulbar projections.

\section{RESULTS}

Morphology of Centrifugal Axons in the Olfactory Bulb Extracellular injections were successfully placed in all subregions of pars principalis. Axons labeled were found in both hemispheres of the $\mathrm{OB}$, and the number and extent of projecttion fibers did not appear qualitatively different based on the location of the injection. Intracellular injections were successful in pars lateralis (four cells) and pars dorsalis (three cells), and both qualitative and quantitative analyses of axons from these cells mirrored the findings from extracellular injection experiments. In coronal sections of the $\mathrm{OB}$, many labeled axons were cut in cross section, but there were a number of fibers whose trajectory ran parallel with the plane of section, and could be followed for great distances in coronal sections. Although these axons could be found in all layers of the olfactory bulb, they appeared most commonly in the granule cell layer (GCL), where they displayed varicosities (presumed en passant synaptic boutons) at fairly regular intervals. These boutons did not appear to target groups of granule cells in the $\mathrm{OB}$, and they did not appear to form clusters (Fig. 1).
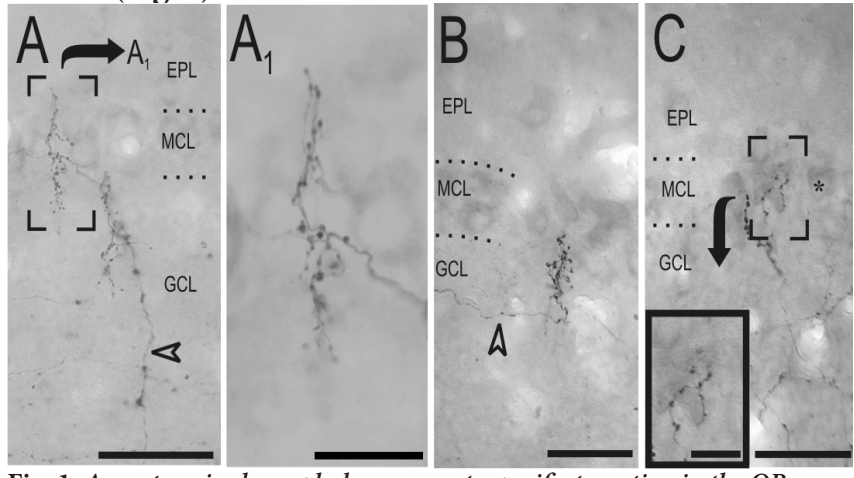

Fig. 1: Axon terminal morphology suggests specific targeting in the $O B$. Axons in the $\mathrm{OB}$ from cells in the AON labeled with Phaseolus vulgaris leucoagglutinin. Labeled axons display columnar orientations (A, B) with dense synaptic boutons in the region around the mitral cell layer (MCL; A, $\left.\mathbf{A}_{1}\right)$. Boutons are relatively sparse on these axons until they orient radially and reach the site of termination (arrowhead in A, B). Other AON cells appeared to target mitral cells preferentially $(\mathbf{C})$, forming a ring of boutons within the MCL. This highly specific targeting by individual axon terminals suggests that the AON is involved in regulating mitral cell activity. Scale bar $=25 \mu \mathrm{m}$ in $\mathbf{A}, \mathbf{B}$ and $\mathbf{C} ; 10 \mu \mathrm{m}$ in $\mathbf{A}_{1}$ and inset of $\mathbf{C}$.

Axons that were found running in the superficial layers of the $\mathrm{OB}$-including the inner plexiform layer (IPL), the mitral cell layer (MCL), the external plexiform layer (EPL), and the glomerular layer (GL)-displayed a different terminal morphology. The most striking difference was that stalked and terminal varicosities that appeared to target individual cells or clusters of cells within a small area in the OB (Fig. 1). Such profiles were seen in all layers of the $\mathrm{OB}$, but were most prevalent in the EPL, MCL and IPL.

Quantification of Centrifugal Axons in the Olfactory Bulb To determine whether certain layers were targeted by centrifugal fibers, we determined the overall length of fibers traced within each layer of the OB (Fig. 2). The highest proportion of 


\section{Brief Report}

corticobulbar axons were found in the GCL (average $=76.6 \%$ of fibers per section $( \pm 1.82 \mathrm{SE})$ ); each of the other layers held fewer than 10 percent of the labeled axons in the OB (GL, 4.38\% \pm 0.39 ; EPL, $8.14 \% \pm 0.33$; MCL, 3.37\% \pm 0.34 ; IPL, $7.85 \% \pm$ $0.65)$. In addition, the GCL displayed the highest proportion of axonal varicosities (presumed synaptic boutons), accounting for nearly $70 \%$ of sampled varicosities $(68.9 \% \pm 1.88)$, with each of the other layers displaying fewer than $10 \%$ of labeled boutons (GL, 6.24\% \pm 0.56; EPL, 6.12\% \pm 0.35; MCL, 4.79\% \pm 0.43 ; IPL, $8.92 \% \pm 0.62)$.

To determine whether boutons were evenly distributed along axons in all layers of the $\mathrm{OB}$, we expressed the proportion of boutons to the proportion of axon length in each layer as a ratio. Thus, if boutons were evenly spaced on axons independent of location, then the proportion of boutons present in any given layer should equal the proportion of axons found in that layer (a ratio of 1.0). As shown in Fig. 2B, the results of this analysis reveal that a significantly greater proportion of boutons per axon length is present in the MCL (average ratio $=2.02 \pm 0.10$ ) and EPL $(2.35 \pm 0.15)$, while the GCL has a relatively low proportion of boutons per axon length $(0.88 \pm .01)$.

\section{DISCUSSION}

Sensory processing is usually considered as a unidirectional process; physical stimuli impinge on specialized sensory receptors, which send a neural signal to the central nervous system and brain, which processes the signal, interprets it, and builds a behavioral response based on the incoming information. However, it has long been known that early relay stations in this process can be influenced by previous or ongoing processing in the brain.

Despite its relatively straightforward laminar structure, the circuitry in the $\mathrm{OB}$ is a complex network of no fewer than seven distinct excitatory and inhibitory cell types that either receive or modulate activity initiated by input from olfactory sensory neurons. Because olfaction is a primary sensory system for rodents, this network is likely to be highly active at almost all times, unless regulated by the extensive network of inhibitory cells. The results of this study demonstrate a mechanism by which cortical processing in the AON may directly modulate principal neuronal activity in the OB. Preliminary examinations with electron microscopy on these projections strongly suggest that the synaptic connection is direct and excitatory, raising the possibility that ongoing olfactory processing in cortex may prime $\mathrm{OB}$ circuitry to fire (or fire more strongly) when a predicted or desirable odor is detected, or based on internal motivational states.

\section{METHODS}

All procedures were performed in accordance with $\mathrm{NIH}$ guidelines under protocols approved by institutional animal care and use committee. The seventeen male Long-Evans rats (280-350 g; Harlan) used for this study were anesthetized with an intraperitoneal injection of anasthetic (ketamine 0.5 $\mathrm{mg} / \mathrm{kg}$; medetomidine hydrochloride $0.32 \mathrm{mg} / \mathrm{kg}$ ) and the head secured in a stereotaxic frame. A small hole was drilled in the skull, a glass pipette was lowered into the brain, and tracer injected iontophoretically by delivering 2.5-5 A of DC current on a $50 \%$ duty cycle for 5-15 minutes. Anesthesia was reversed with atipamezole hydrochloride. 7 to 10 days following the injection, brains were removed, postfixed, cryoprotected, and sections were cut at $40 \mu \mathrm{m}$.
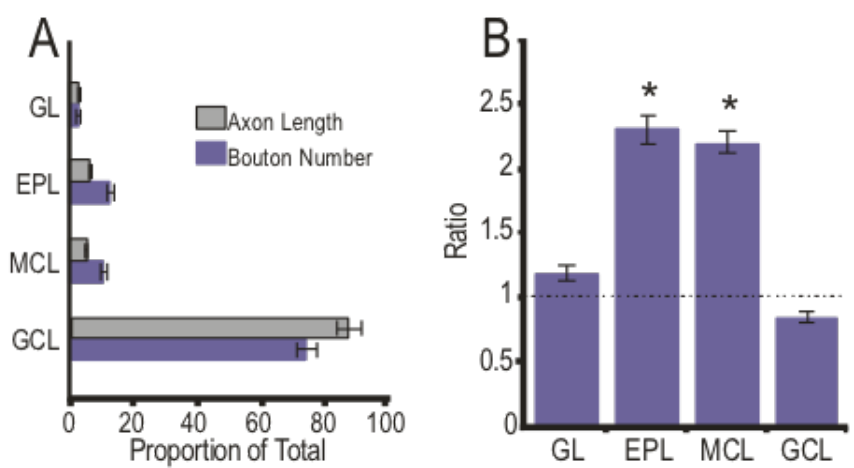

Fig. 2: Quantitative analyses of axon terminals in the olfactory bulb.

A) Proportion of the total axon length (grey) and number of boutons (purple) exhibited by corticofugal projections in each layer of the AON. B) Ratio of the number of boutons found in each layer to the proportion of boutons found in that layer; if bouton spacing is independent of layer, a ratio of 1.0 would be observed. These results demonstrate that although most corticofugal axons travel through the GCL, terminal boutons from AON cells specifically target OB layers containing principle cells (EPL and MCL); ${ }^{*} \mathrm{p}<0.05$ from GL and GCL (Bonferroni corrected t-test).

Immunocytochemistry for PHA-L

Sections were processed for PHA-L immunoreactivity as described previously ${ }^{1}$. Briefly, sections were washed in PBS containing $2 \%$ bovine serum albumin and $0.5 \%$ Triton $\mathrm{X}-100$, followed by incubation with primary antibody overnight, washed and incubated in secondary antibodies, then reacted with avidin. Staining was visualized with $0.04 \%$ 3,3-diaminobenzadine. Sections were dry mounted on gelcoated slides and coverslipped.

Tissue Analysis

Tracings of PHA-L labeled axons were digitized on a computer running Neurolucida software at a final magnification of $200 \mathrm{X}$ to $400 \mathrm{X}$. Features of axonal branching and locations of all varicosities and stalked profiles (presumed synaptic boutons) were included. For quantitative measures, squares $(250$ X $250 \mu \mathrm{m})$ were randomly placed over coronal sections of OB labeled for PHA-L (extracellular tracings), and detailed analysis was carried out at $400 \mathrm{X}$ final magnification, by layer, for every labeled process within the square. Analysis included total axon length, bouton number, location, and inter-bouton distance. Detailed methods at www.Neuro-Cloud.net .

\section{PROGRESS AND COLLABORATIONS}

To see up-to-date progress or if you are interested in contributing to this project visit www.neuro-cloud.net/ nature-precedings/illig2

\section{ACKNOWLEDGEMENTS}

The author thanks Melanie Kok for expert technical assistance. This work was supported by National Institute on Deafness and Communicative Disorders grant DC005557 to KRI.

\section{COMPETING FINANCIAL INTERESTS}

The author declares no competing financial interests.

Submitted online at http://www.precedings.nature.com

1. Illig KR, Eudy J (2009) J Comp Neurol 512:115-123.

2. Meyer EA et al. (2006) J Comp Neurol 498:786-795.

3. Brunjes PC et al. (2005) Brain Res Rev 50:305-335.

4. Lei et al (2006) J Neurosci 15:12023-12032. 